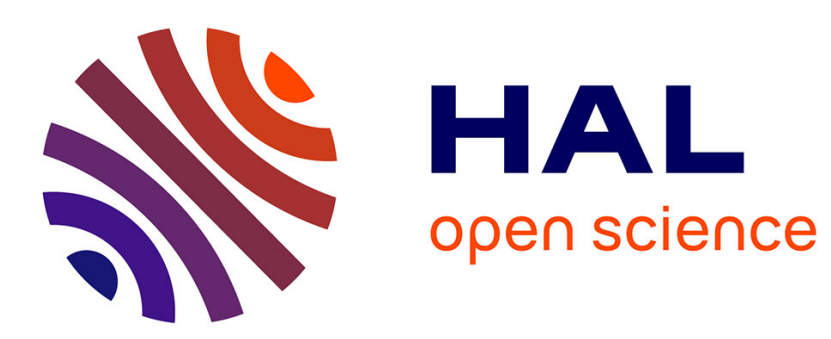

\title{
Decentralized Interference Channels with Noisy Feedback Possess Pareto Optimal Nash Equilibria
}

\author{
Samir M. Perlaza, Ravi Tandon, H. Vincent Poor
}

\section{To cite this version:}

Samir M. Perlaza, Ravi Tandon, H. Vincent Poor. Decentralized Interference Channels with Noisy Feedback Possess Pareto Optimal Nash Equilibria. Proc. of the 6th International Symposium on Communications, Control, and Signal Processing (ISCCSP 2014), May 2014, Athens, Greece. hal00957146

HAL Id: hal-00957146

\section{https://hal.inria.fr/hal-00957146}

Submitted on 10 Mar 2014

HAL is a multi-disciplinary open access archive for the deposit and dissemination of scientific research documents, whether they are published or not. The documents may come from teaching and research institutions in France or abroad, or from public or private research centers.
L'archive ouverte pluridisciplinaire HAL, est destinée au dépôt et à la diffusion de documents scientifiques de niveau recherche, publiés ou non, émanant des établissements d'enseignement et de recherche français ou étrangers, des laboratoires publics ou privés. 


\title{
Decentralized Interference Channels with Noisy Feedback Possess Pareto Optimal Nash Equilibria
}

\author{
Samir M. Perlaza* ${ }^{*}$, Ravi Tandon ${ }^{\dagger}$ and H. Vincent Poor ${ }^{\ddagger}$ \\ * CITI Laboratory. INRIA - Rhône Alpes, Villeurbanne, France \\ $\dagger$ Hume Center and Department of ECE, Virginia Tech, Blacksburg, VA \\ $\ddagger$ Department of Electrical Engineering. Princeton University, Princeton, NJ \\ samir.perlaza@inria.fr, tandonr@vt.edu, poor@princeton.edu
}

\begin{abstract}
In this paper, the price of anarchy (PoA) and the price of stability (PoS) of a game arising in a two-user decentralized interference channel (DIC) with noisy feedback in which transmit-receiver pairs seek an optimal individual transmission rate are fully characterized. In particular, it is shown that in all interference regimes, there always exists at least one Pareto optimal Nash equilibrium (NE). More specifically, there always exists an NE at which players maximize the network sum-rate and thus, the PoS of the corresponding game is always equal to one. A second result provides closed form expressions for the PoA, which allows the full characterization of the reduction of the sum rate due to the anarchic behavior of all transmitterreceiver pairs.
\end{abstract}

\section{INTRODUCTION}

The use of perfect output feedback has been recently shown to be highly beneficial in the two-user decentralized interference channel (DIC) [1], i.e., an interference channel in which transmit-receiver pairs autonomously tune their own transmit/receive configurations seeking an optimal individual transmission rate [2]. Even in the case of noisy feedback, these benefits have been shown to be very relevant. For instance, the set of achievable rate pairs at a Nash equilibrium (NE), also known as the Nash region, has been shown to be enlarged, in most of the cases, thanks to the use of feedback despite the existence of noise [3].

In this paper, tools from game theory, namely the price of anarchy (PoA) [4] and the price of stability (PoS) [5] are used to study the efficiency of the NEs in the linear deterministic DIC (LD-DIC) with noisy feedback. The PoA and the PoS are both measures of the efficiency of the set of equilibria of any given game. Basically, the PoA measures the loss of global performance due to decentralization by comparing the maximum sum utility achieved under global control with the minimum sum utility achieved at the Nash equilibrium. The PoS measures the loss of global performance due to decentralization by comparing the maximum sum utility achieved under the global control with the maximum sum utility achieved at the Nash equilibrium.

The main contributions of this paper are closed form expressions for both the PoA and the PoS. Scenarios in which the use of feedback leads to Pareto optimal NEs are identified and interesting conclusions regarding the worst NE scenarios are drawn for the different interference regimes. In particular, it is shown that if players are equipped with equilibrium selection capabilities (see discussions in [6]), no loss in the sum rate

This research was supported in part by the Air Force Office of Scientific Research under MURI Grant FA9550-09-1-0643 and in part by the Marie Curie Outgoing Fellowship Program under Award No. FP7-PEOPLE-IOF2011-298532. is observed due to the anarchical behavior of all network components.

\section{Linear Deterministic IC With NoISy FeEDBACK}

Consider a two-user Gaussian DIC with noisy feedback [3]. Transmitter $i$, with $i \in\{1,2\}$, communicates with receiver $i$ during $T$ consecutive blocks subject to the interference produced by transmitter $j \in\{1,2\} \backslash\{i\}$. The linear deterministic approximation [7] of this decentralized channel, known as two-user LD-DIC with noisy feedback, can be described by six parameters: $\vec{n}_{11}, \vec{n}_{22}, n_{12}, n_{21}$, $\overleftarrow{n}_{11}$ and $\overleftarrow{n}_{22}$. The parameter $\vec{n}_{i i}$ captures the signal strength from transmitter $i$ to receiver $i ; n_{i j}$ captures the interference strength from transmitter $j$ to receiver $i$; and $\overleftarrow{n}_{i i}$ captures the feedback signal strength from receiver $i$ to transmitter $i$. Let $q=\max _{(i, j) \in\{1,2\}^{2}} \max \left(n_{i j}, \overleftarrow{n}_{i i} \vec{n}_{i i}\right)$ be strictly positive and finite. Let also $\mathcal{X}_{i} \subset\{0,1\}^{q}$ be the codebook of the receivertransmitter pair $i$. Then, the input-output relationship of the two user LD-DIC is

$$
\begin{aligned}
& \overrightarrow{\boldsymbol{y}}_{1}^{(t)}=\boldsymbol{S}^{q-\vec{n}_{11}} \boldsymbol{x}_{1}^{(t)}+\boldsymbol{S}^{q-n_{12}} \boldsymbol{x}_{2}^{(t)}, \text { and } \\
& \overrightarrow{\boldsymbol{y}}_{2}^{(t)}=\boldsymbol{S}^{q-n_{21}} \boldsymbol{x}_{1}^{(t)}+\boldsymbol{S}^{q-\vec{n}_{22}} \boldsymbol{x}_{2}^{(t)},
\end{aligned}
$$

where $\boldsymbol{x}_{i}^{(t)}=\left(x_{i, 1}^{(t)}, \ldots, x_{i, N_{i}}^{(t)}\right)^{T} \in \mathcal{X}_{i}$ is the channel input vector generated by transmitter $i$ and $\boldsymbol{y}_{i}^{(t)}=\left(y_{i, 1}^{(t)}, \ldots, y_{i, q}^{(t)}\right)^{T} \in$ $\{0,1\}^{q}$ is the channel output received by receiver $i$ during block $t \in\{1, \ldots, T\}$. Therefore, the code-length $N_{i}$, in the LD-DIC, satisfies the condition that $N_{i} \leqslant q$. The matrix $\boldsymbol{S}$ is a $q \times q$ lower shift matrix and all additions and multiplications are over a binary field.

A noisy feedback link from receiver $i$ to transmitter $i$ allows at the end of each block $t$ the observation of the output $\overrightarrow{\boldsymbol{y}}_{i}^{(t-d)}$ at transmitter $i$, within a finite delay of $d$ blocks. Thus, the feedback signal available at transmitter $i$ during block $t$ is

$$
\overleftarrow{\boldsymbol{y}}_{i}^{(t)}=\boldsymbol{S}^{q-\overleftarrow{n}_{i i}} \overrightarrow{\boldsymbol{y}}_{i}^{(t-d)}
$$

Let $M_{i}$ be the number of information bits $b_{i, 1}^{(t)}, \ldots, b_{i, M_{i}}^{(t)}$ sent by transmitter $i$ at every block $t$. Hence, the encoder of transmitter $i$, during block $t>d$, can be modeled as a deterministic mapping $f_{i}^{(t)}:\left\{1, \ldots, 2^{M_{i}}\right\} \times\{0,1\}^{t-d} \rightarrow \mathcal{X}_{i}$ such that $\boldsymbol{x}_{i}^{(t)}=f_{i}^{(t)}\left(k, \overleftarrow{\boldsymbol{y}}_{i}^{(1)}, \ldots, \overleftarrow{\boldsymbol{y}}_{i}^{(t-d)}\right) \in \mathcal{X}_{i}$, where $k$ is the index of the message to be transmitted. Note that for blocks for which $t \leqslant d$, the encoder is a mapping $f_{i}^{(t)}:\left\{1, \ldots, 2^{M_{i}}\right\} \rightarrow$ $\mathcal{X}_{i}$ for which the symbols $\boldsymbol{x}_{i}^{(t)}=f_{i}^{(t)}(k)$ do not depend on the feedback signals $\overleftarrow{\boldsymbol{y}}_{i}^{(1)}, \ldots, \overleftarrow{\boldsymbol{y}}_{i}^{(t-d)}$. At the end of 
the complete transmission, after block $T$, receiver $i$ uses the sequences $\overrightarrow{\boldsymbol{y}}_{i}^{(1)}, \ldots, \overrightarrow{\boldsymbol{y}}_{i}^{(T)}$ to generate estimates $\hat{b}_{i, \ell}^{(t)}$ of the transmitted bits $b_{i, \ell}^{(t)}, \forall(\ell, t) \in\left\{1, \ldots, M_{i}\right\} \times\{1, \ldots, T\}$. The average bit error probability of transmitter $i$ during block $t$, denoted by $p_{i}^{(t)}$, is calculated as follows:

$$
p_{i}^{(t)}=\frac{1}{M_{i}} \sum_{\ell=1}^{M_{i}} \mathbb{1}_{\left\{\hat{b}_{i, \ell}^{(t)} \neq b_{i, \ell}^{(t)}\right\}} .
$$

The rate pair $\left(R_{1}, R_{2}\right) \in \mathbb{R}_{+}^{2}$ is achievable if there exists at least one pair of codebooks $\mathcal{X}_{1}$ and $\mathcal{X}_{2}$ with codewords of length $N_{1}$ and $N_{2}$, respectively, with the corresponding encoding functions $f_{1}$ and $f_{2}$ such that the average bit error probability can be made arbitrarily small by letting the block lengths $N_{1}$ and $N_{2}$ grow to infinity.

The aim of transmitter $i$ is to autonomously choose its transmit configuration $s_{i}$ in order to maximize its achievable rate $R_{i}$. More specifically, the transmit configuration $s_{i}$ can be described in terms of the number of information bits per block $M_{i}$, the block length $N_{i}$, the codebook $\mathcal{X}_{i}$, the encoding functions $f_{i}$, etc. Note that the rate achieved by receiver $i$ depends on both configurations $s_{1}$ and $s_{2}$ due to the mutual interference naturally arising in the DIC. This reveals the competitive interaction between both links in the LD-DIC and justifies the use of tools from game theory in its analysis.

\section{A. Game Formulation}

The competitive interaction through mutual interference between the transmitters in the two-user DIC can be modeled by the following game in normal-form:

$$
\mathcal{G}=\left(\mathcal{K},\left\{\mathcal{A}_{k}\right\}_{k \in \mathcal{K}},\left\{u_{k}\right\}_{k \in \mathcal{K}}\right) .
$$

The set $\mathcal{K}=\{1,2\}$ is the set of players, that is, the set of transmitter-receiver pairs. The sets $\mathcal{A}_{1}$ and $\mathcal{A}_{2}$ are the sets of actions of players 1 and 2, respectively. An action of player $i$, which is denoted by $s_{i} \in \mathcal{A}_{i}$, is basically its transmit/receive configuration as described above. The utility function of player $i$ is $u_{i}: \mathcal{A}_{1} \times \mathcal{A}_{2} \rightarrow \mathbb{R}_{+}$and it is defined as the achieved rate of transmitter $i$, that is,

$$
u_{i}\left(s_{1}, s_{2}\right)= \begin{cases}R_{i}\left(s_{1}, s_{2}\right), & \text { if } \quad \forall t \in\{1, \ldots, T\}, p_{i}^{(t)}<\epsilon, \\ 0, & \text { otherwise }\end{cases}
$$

where $\epsilon>0$ is an arbitrarily small number and $R_{i}\left(s_{1}, s_{2}\right)$ denotes a transmission rate achievable with the configurations $s_{1}$ and $s_{2}$ such that $p_{i}^{(t)}<\epsilon$. Often, the rate $R_{i}\left(s_{1}, s_{2}\right)$ is written as $R_{i}$ for the sake of simplicity.

Some action profiles $s=\left(s_{1}, s_{2}\right) \in \mathcal{A}_{1} \times \mathcal{A}_{2}$ are particularly important in the analysis of this game. These actions profiles are referred to as $\eta$-Nash equilibria $(\eta$-NE) and obey the following definition:

Definition 1 ( $\eta$-Nash equilibrium): In the game $\mathcal{G}=$ $\left(\mathcal{K},\left\{\mathcal{A}_{k}\right\}_{k \in \mathcal{K}},\left\{u_{k}\right\}_{k \in \mathcal{K}}\right)$, an action profile $\left(s_{1}^{*}, s_{2}^{*}\right)$ is an $\eta$ Nash equilibrium, with $\eta \geqslant 0$, if $\forall i \in \mathcal{K}$ and $\forall s_{i} \in \mathcal{A}_{i}$, $u_{i}\left(s_{i}, s_{j}^{*}\right) \leqslant u_{i}\left(s_{i}^{*}, s_{j}^{*}\right)+\eta$.

From Def. 1, it becomes clear that if $\left(s_{1}^{*}, s_{2}^{*}\right)$ is an $\eta$-Nash equilibrium, then none of the transmitters can increase its own transmission rate more than $\eta$ bits per block by changing its own transmit configuration and keeping the average bit error probability arbitrarily close to zero. Thus, at a given $\eta$-NE, every transmitter achieves a utility (transmission rate) that is $\eta$-close to its maximum achievable rate given the transmit configuration of the other transmitter. Note that if $\eta=0$, then the classical definition of Nash equilibrium is obtained. The relevance of the notion of equilibrium is that at any NE, every transmitter configuration is optimal with respect to the configuration of the other transmitters.

The set of rate pairs achieved at an NE is known as the Nash region.

Definition 2 (Nash Region): An achievable rate pair $\left(R_{1}, R_{2}\right)$ is said to be in the Nash region of the game $\mathcal{G}=\left(\mathcal{K},\left\{\mathcal{A}_{k}\right\}_{k \in \mathcal{K}},\left\{u_{k}\right\}_{k \in \mathcal{K}}\right)$ if there exists an action profile $\left(s_{1}^{*}, s_{2}^{*}\right)$ that is an $\eta$-Nash equilibrium for an arbitrarily small $\eta$ and the following holds $u_{1}\left(s_{1}^{*}, s_{2}^{*}\right)=R_{1}$ and $u_{2}\left(s_{1}^{*}, s_{2}^{*}\right)=$ $R_{2}$.

B. Capacity and Nash Region of the Symmetric LD-DIC with Noisy Feedback

A particular case of the LD-DIC is the symmetric case. That is, an LD-DIC in which $\vec{n}=\vec{n}_{11}=\vec{n}_{22}, m=n_{12}=$ $n_{21}$ and $\overleftarrow{n}=\overleftarrow{n}_{11}=\overleftarrow{n}_{22}$. The capacity region of the twouser symmetric LD-DIC with noisy feedback is denoted by $\mathcal{C}_{(\vec{n}, \overleftarrow{n}, m)}$ and it is fully characterized by Theorem 1 in [8].

Lemma 1 (Theorem 1 in [8]): The capacity region $\mathcal{C}_{(\vec{n}, \overleftarrow{n}, m)}$ of the two-user LD-DIC with noisy feedback corresponds to the set of non-negative rate pairs $\left(R_{1}, R_{2}\right)$ that satisfy for all $i \in\{1,2\}$

$$
\begin{gathered}
R_{i} \leqslant \max (\vec{n}, m) \\
R_{i} \leqslant \vec{n}+(\overleftarrow{n}-\vec{n})^{+}, \\
R_{1}+R_{2} \leqslant(\vec{n}-m)^{+}+\max (\vec{n}, m) \\
R_{1}+R_{2} \leqslant 2 \max \left((\vec{n}-m)^{+}, m\right) \\
+2 \min \left((\vec{n}-m)^{+},\left(\overleftarrow{n}-\max \left((\vec{n}-m)^{+}, m\right)\right)^{+}\right) \\
R_{1}+2 R_{2} \leqslant(\vec{n}-m)^{+}+\max (\vec{n}, m) \\
+\min \left((\vec{n}-m)^{+},\left(\overleftarrow{n}-\max \left((\vec{n}-m)^{+}, m\right)\right)^{+}\right) \\
+\max \left((\vec{n}-m)^{+}, m\right) \\
2 R_{1}+R_{2} \leqslant(\vec{n}-m)^{+}+\max (\vec{n}, m) \\
+\min \left((\vec{n}-m)^{+},\left(\overleftarrow{n}-\max \left((\vec{n}-m)^{+}, m\right)\right)^{+}\right) \\
+\max \left((\vec{n}-m)^{+}, m\right) .
\end{gathered}
$$

The NE region (Def. 2) of the symmetric LD-DIC with noisy feedback with parameters $\vec{n}, \overleftarrow{n}$ and $m$ is given by

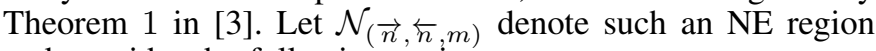
and consider the following region:

$$
\mathcal{B}_{(\vec{n}, \overleftarrow{n}, m)}=\left\{\left(R_{1}, R_{2}\right): L \leqslant R_{i} \leqslant U, \forall i \in\{1,2\}\right\},
$$

where $L$ and $U$ are defined as follows:

$$
\begin{gathered}
L=(\vec{n}-m)^{+} \text {and } \\
U=\left\{\begin{array}{lll}
U^{(a)} & \text { if } & m \geqslant \vec{n} \\
U^{(b)} & \text { if } & m \leqslant \vec{n}
\end{array},\right.
\end{gathered}
$$

with $\quad U^{(a)} \quad=\quad \min (\max (\vec{n}, \overleftarrow{n}), m) \quad$ and $U^{(b)} \quad=\max (\vec{n}, m)-\min \left((\vec{n}-m)^{+}, m\right)+$ 
$\left(\min \left((\vec{n}-m)^{+}, m\right)-(\max (\vec{n}, m)-\overleftarrow{n})\right)^{+}$

Hence, following this notation, Theorem 1 in [3] can be written as follows:

Lemma 2: [Theorem 1 in [3]] The Nash region of the twouser symmetric LD-DIC with noisy feedback, with parameters $\vec{n}, m$ and $\overleftarrow{n}$, is $\mathcal{N}_{(\vec{n}, \overleftarrow{n}, m)}=\mathcal{B}_{(\vec{n}, \overleftarrow{n}, m)} \cap \mathcal{C}_{(\vec{n}, \overleftarrow{n}, m)}$

\section{Main Results}

In this section, the efficiency of the NEs of the game $\mathcal{G}$ is studied. To this end, let $\mathcal{A}=\mathcal{A}_{1} \times \mathcal{A}_{2}$ be the set of all possible action profiles, $\mathcal{A}_{\mathrm{NE}} \subset \mathcal{A}$ be the set of $\mathrm{NE}$ action profiles and consider the following lemma.

Lemma 3 (Maximum Sum-Rate at an NE): For $(\vec{n}, \overleftarrow{n}, m) \in \mathbb{N}^{3}$ let $\vec{\Sigma}(,, \pi$, all equality:

$$
\begin{aligned}
\bar{\Sigma}_{(\vec{n}, \overleftarrow{n}, m)}=\max & R_{1}\left(s_{1}, s_{2}\right)+R_{2}\left(s_{1}, s_{2}\right) \\
\text { s.t. } & \left(s_{1}, s_{2}\right) \in \mathcal{A} \\
& \left(R_{1}\left(s_{1}, s_{2}\right), R_{2}\left(s_{1}, s_{2}\right)\right) \in \mathcal{C}_{(\vec{n}, \overleftarrow{n}, m)} .
\end{aligned}
$$

Then, there always exists at least one rate pair $\left(R_{1}^{*}, R_{2}^{*}\right) \in$ $\mathcal{N}_{(\vec{n}, \overleftarrow{n}, m)}$ that satisfies $\bar{\Sigma}_{(\vec{n}, \overleftarrow{n}, m)}=R_{1}^{*}+R_{2}^{*}$ and

$$
\begin{aligned}
& \bar{\Sigma}_{(\vec{n}, \overleftarrow{n}, m)} \\
& =\min \left\{(\vec{n}-m)^{+}+\max (\vec{n}, m), 2 \max \left((\vec{n}-m)^{+}, m\right)\right. \\
& \left.+2 \min \left((\vec{n}-m)^{+},\left(\overleftarrow{n}-\max \left((\vec{n}-m)^{+}, m\right)\right)^{+}\right)\right\} .
\end{aligned}
$$

Basically, Lemma 3 states that there always exists at least one $\mathrm{NE}$ that maximizes the sum rate. A formal proof of Lemma 3 is not presented in this paper due to space constraints. However, this proof comprises two parts. In the first part, it is shown by direct verification that for all $(\vec{n}, \overleftarrow{n}, m) \in \mathbb{N}^{3}$, there always exists at least one rate pair $\left(R_{1}^{*}, R_{2}^{*}\right) \in \mathcal{C}_{(\vec{n}, \overleftarrow{n}, m)}$ that saturates either (8) or (9). This proves that $\left(R_{1}^{*}, R_{2}^{*}\right)$ is in the boundary of the capacity region, that is, $\bar{\Sigma}_{(\vec{n}, \overleftarrow{n}, m)}=R_{1}^{*}+R_{2}^{*}$. In the second part, it is shown that $\left(R_{1}^{*}, R_{2}^{*}\right) \in \mathcal{B}_{(\vec{n}, \overleftarrow{n}, m)}$. This shows that the rate pair $\left(R_{1}^{*}, R_{2}^{*}\right)$ is achieved at an NE, which completes the proof.

Consider also the following lemma.

Lemma 4 (Minimum Sum-Rate at an NE): For all $(\vec{n}, \overleftarrow{n}, m) \in \mathbb{N}^{3}$, let $\underline{\Sigma}_{(\vec{n}, \overleftarrow{n}, m)}$ satisfy the following equality:

$$
\begin{aligned}
\underline{\Sigma}_{(\vec{n}, \overleftarrow{n}, m)}=\min & R_{1}\left(s_{1}, s_{2}\right)+R_{2}\left(s_{1}, s_{2}\right) \\
\text { s.t. } & \left(s_{1}, s_{2}\right) \in \mathcal{A}_{\mathrm{NE}} \\
& \left(R_{1}\left(s_{1}, s_{2}\right), R_{2}\left(s_{1}, s_{2}\right)\right) \in \mathcal{C}_{(\vec{n}, \overleftarrow{n}, m)} .
\end{aligned}
$$

Then, there always exists at least one rate pair $\left(R_{1}^{*}, R_{2}^{*}\right) \in$ $\mathcal{N}_{(\vec{n}, \overleftarrow{n}, m)}$ that satisfies $\underline{\Sigma}_{(\vec{n}, \overleftarrow{n}, m)}=R_{1}^{*}+R_{2}^{*}$, with $\underline{\Sigma}_{(\vec{n}, \overleftarrow{n}, m)}=2(\vec{n}-m)$.

Lemma 4 provides a lower bound for the sum rate at an NE. The proof of Lemma 4 follows from Lemma 2.

From Lemma 3 and Lemma 4, the sum rate at any NE can be lower and upper bounded. Using these results, closed-form expressions can be provided for the PoA and the PoS of the game $\mathcal{G}$.

\section{A. Price of Anarchy}

From its definition in [4], it holds that the PoA of the game $\mathcal{G}$ is

$$
\operatorname{PoA}(\mathcal{G})=\frac{\max _{\left(s_{1}, s_{2}\right) \in \mathcal{A}} \sum_{i=1}^{2} R_{i}\left(s_{1}, s_{2}\right)}{\min _{\left(s_{1}^{*}, s_{2}^{*}\right) \in \mathcal{A}_{\mathrm{NE}}} \sum_{i=1}^{2} R_{i}\left(s_{1}^{*}, s_{2}^{*}\right)} .
$$

From Lemma 3 and Lemma 4, the following proposition holds.

Proposition 1 (PoA): The PoA in the game $\mathcal{G}$ of the symmetric LD-DIC with noisy feedback with parameters $\vec{n}, m$ and $\overleftarrow{n}$ satisfies the following equality:

$$
\operatorname{PoA}(\mathcal{G})=\left\{\begin{array}{lll}
\frac{\bar{\Sigma}_{(\vec{n}, \widehat{r}, m)}}{2(\vec{n}-m)^{+}} & \text {if } & \vec{n}>m \\
\infty & \text { if } & \vec{n} \leqslant m .
\end{array}\right.
$$

The relevance of Proposition 1 is highlighted by the following corollaries that describe the PoA in particular interference regimes. Consider the following parameters $\alpha=\frac{m}{\vec{n}}$ and $\beta=\frac{\overleftarrow{n}}{\bar{n}}$.

1) Very Weak Interference Regime: The PoA of the game $\mathcal{G}$ in the very weak interference regime $\left(\alpha \leqslant \frac{1}{2}\right)$ is fully characterized by the following corollary of Proposition 1.

Corollary 1 (PoA in Very Weak Interference Regime):

The PoA in the game $\mathcal{G}$ of the symmetric $L D-D I C$ with noisy feedback in the very weak interference regime $\left(\alpha \leqslant \frac{1}{2}\right)$ is

$$
\operatorname{PoA}(\mathcal{G})= \begin{cases}1 & \text { if } \beta \leqslant 1-\alpha \\ \min \left(\frac{\beta}{1-\alpha}, \frac{2-\alpha}{2(1-\alpha)}\right) & \text { if } 1-\alpha \leqslant \beta \leqslant 1 \\ \frac{2-\alpha}{2(1-\alpha)} & \text { if } \beta \geqslant 1-\alpha .\end{cases}
$$

Corollary 1 leads to an interesting upper bound for the PoA in the very weak interference regime: $1 \leqslant \operatorname{PoA}(\mathcal{G}) \leqslant \frac{3}{2}$. Note that no loss in the sum rate is observed due to the anarchical behavior of the players $(\mathrm{PoA}(\mathcal{G})=1$ ) when the signal to noise ratios (SNRs) of the feedback links are lower than the signal to interference ratios (SIRs) of the direct links, i.e., $\beta \leqslant 1-\alpha$ (see Fig. 1). This is explained by the fact that under these conditions, $\alpha \leqslant \frac{1}{2}$ and $\beta \leqslant 1-\alpha$, the Nash regions with and without feedback links are identical and thus, the unique rate pair achievable at an NE, which is achieved by treating interference as noise, falls on the boundary of the capacity region [1], [2]. Alternatively, when the SNRs of the feedback links are higher than the SIRs of the direct links $\beta \geqslant 1-\alpha$, the use of feedback enlarges the NE region and thus, treating interference as noise becomes the worse NE [3]. Therefore, it follows that $\operatorname{PoA}(\mathcal{G})>1$ (see Fig. 1). The upperbound PoA $(\mathcal{G}) \leqslant \frac{3}{2}$ follows from the fact that increasing the SNRs of the feedback links beyond the SIRs of the direct links does not push forward the sum-rate upper bounds of the capacity region [3] and for all $\alpha \leqslant \frac{1}{2}$, it follows that $\frac{2-\alpha}{2(1-\alpha)} \leqslant \frac{3}{2}$ (see Fig. 1). That is, the sum rate at the worst equilibrium can be at most the highest achievable sum-rate in a fully centralized system but it is never less than $\frac{2}{3}$ of the highest achievable sum-rate in a fully centralized system.

2) Weak Interference Regime: The PoA of the game $\mathcal{G}$ in the weak interference regime $\left(\frac{1}{2} \leqslant \alpha \leqslant \frac{2}{3}\right)$ is fully characterized by the following corollary of Proposition 1 .

Corollary 2 (PoA in Weak Interference Regime): The PoA in the game $\mathcal{G}$ of the symmetric LD-DIC with noisy feedback 


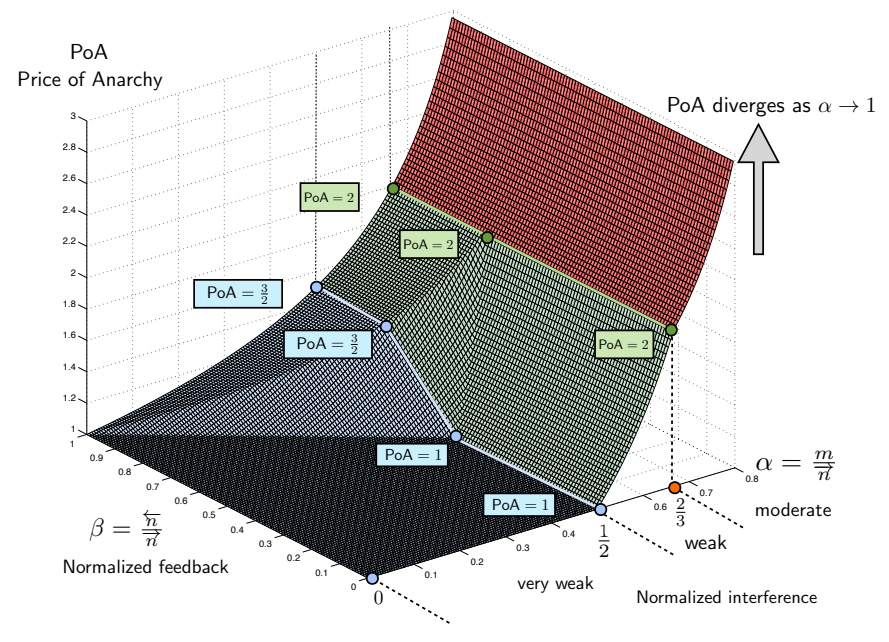

Fig. 1. PoA as a function of the parameters $\alpha$ and $\beta$.

in the weak interference regime $\left(\frac{1}{2} \leqslant \alpha \leqslant \frac{2}{3}\right)$ is

$$
\operatorname{PoA}(\mathcal{G})= \begin{cases}\frac{\alpha}{1-\alpha} & \text { if } \beta \leqslant \alpha \\ \min \left(\frac{\beta}{1-\alpha}, \frac{2-\alpha}{2(1-\alpha)}\right) & \text { if } \quad \alpha \leqslant \beta \leqslant 1 \\ \frac{2-\alpha}{2(1-\alpha)} & \text { if } \beta \geqslant 1 .\end{cases}
$$

Corollary 2 leads to the following inequalities for the PoA: $1 \leqslant \operatorname{PoA}(\mathcal{G}) \leqslant 2$. Note that in this interference regime, the equality PoA $=1$ holds only when $\alpha=\frac{1}{2}$ and $\beta \leqslant \alpha$ for the reasons explained in the previous sections. For all other values of $\alpha$ satisfying $\frac{1}{2}<\alpha \leqslant \frac{2}{3}$, strict inequality holds, PoA $>1$ (see Fig. 1). This implies that there always exists a reduction of the sum rate when the system becomes decentralized. However, at the worst NE, given that $\operatorname{PoA}(\mathcal{G}) \leqslant 2$, the sum rate is never less than half of the highest sum rate of a fully centralized system.

3) Moderate Interference Regime: The PoA of the game $\mathcal{G}$ in the weak interference regime $\left(\frac{2}{3} \leqslant \alpha \leqslant 1\right)$ is fully characterized by the following corollary of Proposition 1 .

Corollary 3 (PoA in Moderate Interference Regime): The $P o A$ in the game $\mathcal{G}$ of the symmetric LD-DIC with noisy feedback in the weak interference regime $\left(\frac{2}{3} \leqslant \alpha \leqslant 1\right)$ is

$$
\operatorname{PoA}(\mathcal{G})=\frac{2-\alpha}{2(1-\alpha)} .
$$

Corollary 3 leads to the following upper bound for the PoA in the very weak interference regime: $2 \leqslant \operatorname{PoA}(\mathcal{G}) \leqslant \infty$. Therefore, at the worst NE, the sum rate can be at most $\frac{1}{2}$ of the highest sum rate of a fully centralized system. More importantly, in this regime, the PoA is independent of $\beta$. This is basically because the use of feedback enlarges the NE region but does not shift forward the sum rate bounds of $\mathcal{C}_{(\vec{n}, \overleftarrow{n}, m)}$ [8]. Finally note that when $\alpha=1$, none of the transmitterreceiver links is able to transmit at a strictly positive rate at the worst NE, i.e., $\Sigma=0$. Hence, $\operatorname{PoA}(\mathcal{G})$ becomes infinity when $\alpha$ approaches 1 .

4) Strong Interference Regime: The PoA of the game $\mathcal{G}$ in the strong interference regime $(\alpha \geqslant 1)$ grows unboundedly towards infinity. As in the previous case, this is basically due to the fact that $\underline{\Sigma}=0$.

\section{B. Price of Stability}

From its definition in [5], the PoS of the game $\mathcal{G}$ can be written as follows:

$$
\operatorname{PoS}(\mathcal{G})=\frac{\max _{\left(s_{1}, s_{2}\right) \in \mathcal{A}} \sum_{i=1}^{2} R_{i}\left(s_{1}, s_{2}\right)}{\max _{\left(s_{1}^{*}, s_{2}^{*}\right) \in \mathcal{A}_{\mathrm{NE}}} \sum_{i=1}^{2} R_{i}\left(s_{1}^{*}, s_{2}^{*}\right)} .
$$

The following proposition characterizes the PoS of the symmetric LD-DIC with noisy feedback.

Proposition 2 (PoS): The PoS in the game $\mathcal{G}$ of the symmetric LD-DIC with noisy feedback with parameters $\vec{n}, m$ and $\overleftarrow{n}$ is $\operatorname{PoS}(\mathcal{G})=1$, for all $(\vec{n}, \overleftarrow{n}, m) \in \mathbb{N}^{3}$

The proof of Proposition 2 is immediate from Lemma 3.

The price of stability is equal to one, independently of the parameters $\vec{n}, \overleftarrow{n}, m$. This implies that, despite the anarchical behavior of both links, it is always possible to observe an $\mathrm{NE}$ in which no reduction of the sum rate is observed. This observation reveals the tremendous importance of techniques aiming to allow players to choose the equilibrium they would play. An interesting discussion about equilibrium selection is presented in [6].

\section{CONCLUSION}

In this paper, the PoA and the PoS of a game in which transmit-receiver pairs of a DIC with noisy feedback autonomously tune their own transmit/receive configurations seeking an optimal individual transmission rate are fully characterized. In particular, it is shown that in all interference regimes, there always exists at least one Pareto optimal NE and thus, the PoS of the corresponding game is always equal to one. The analysis of the PoA shows that there exist scenarios in which there is no reduction of the sum rate due to the decentralization of the network, mainly in the very weak interference regime. In other scenarios, mainly in the strong interference regime, the loss on the sum rate due to decentralization is unfortunately dramatic.

\section{REFERENCES}

[1] S. M. Perlaza, R. Tandon, H. V. Poor, and Z. Han, "Perfect output feedback in the two-user decentralized interference channel," (Submitted to) IEEE Transactions on Information Theory, Jun. 2013. http://arxiv.org/abs/1306.2878.

[2] R. A. Berry and D. N. C. Tse, "Shannon meets Nash on the interference channel," IEEE Transactions on Information Theory, vol. 57, no. 5, pp. 2821-2836, May 2011.

[3] S. M. Perlaza, R. Tandon, and H. V. Poor, "Symmetric decentralized interference channels with noisy feedback," (Submitted to) IEEE Intl. Symposium on Information Theory (ISIT), Honolulu, HI, USA, Jun. 2014.

[4] E. Koutsoupias and C. Papadimitriou, "Worst-case equilibria," in Proc. 16th Annual Symposium on Theoretical Aspects of Computer Science, Trier, Germany, Mar. 1999.

[5] E. Anshelevich, A. Dasgupta, E. Tardos, and T. Wexler, "Near-optimal network design with selfish agents," in Proc. Annual ACM Symposium on Theory of Computing, San Diego, CA, USA, Jun. 2003.

[6] S. M. Perlaza, S. Lasaulce, H. V. Poor, and Z. Han, "Selecting and learning Nash equilibria via maximum entropy principle," (Submitted to) IEEE Intl. Symposium on Information Theory (ISIT), Honolulu, HI, USA, Jun. 2014.

[7] S. Avestimehr, S. Diggavi, and D. N. C. Tse, "Wireless network information flow: A deterministic approach," IEEE Transactions on Information Theory, vol. 57, no. 4, pp. 1872-1905, Apr. 2011.

[8] S.-Q. Le, R. Tandon, M. Motani, and H. V. Poor, "Approximate capacity region for the symmetric Gaussian interference channel with noisy feedback," (Submitted to) IEEE Transactions on Information Theory, Dec. 2012. http://www.ece.vt.edu/tandonr/IC-Partial-Feedback.pdf. 